\title{
Prospect of wireless body area network technology
}

HUANG Rui

Engineer Training Center

Shanghai Second Polytechnic University

Shanghai China

huangrui@sspu.edu.cn

Abstract : Wireless body area network $(W B A N)$ is one of the important means of personal information collection and transmission, has important practical significance and the prospect of industrialization, has attracted wide attention of industry, academia, and standardization organization .This paper summarized the present situation of research on Key Technologies of WBAN, including low power technology, security technology and quality of service (QoS), and prospected research and development of $W B A N$ in the future.

Keywords-WBAN; WBAN industrialization; WBAN standards; Low power technology

\section{INTRODUCTION}

The aging problem of the world is becoming more and more serious, along with the number of chronic patients rapidly increases, and gradually become a major threat to the health of residents. It is universal concern problem how to effective real-timely monitor the aging population's physical condition in order to achieve the effective for chronic disease prevention and management. The emergence of wireless body area network (WBAN) is expected to ease this problem.

WBAN is a wireless networking technology, based on Radio Frequency (RF) that interconnects a number of small nodes with sensor or actuator capabilities. These nodes operate in close vicinity to, on or few $\mathrm{cm}$ inside a human body, to support various medical area and non-medical area applications ${ }^{[1]}$. For now, WBAN is still in its early stages, and it is faced with a series of challenges in the aspects of low power consumption, network interoperability, system equipment, data security, sensor implementation and so on $^{[2]}$.

\section{RESEARCH STATUS OF WBAN}

WBAN is a new type of network technology, which combines wireless communication technology, sensor technology, network technology and so on, It has great application value in the health field. However, WBAN node design must be in accordance with the requirements of low power consumption, security, and high quality of service (QoS). Because WBAN is health oriented, data transmission must have security, high QoS characteristics, in order to reduce the leakage of personal privacy, to ensure data transmission reliability.

\section{A. Low Power Technology}

Low power consumption is contribute to extend the life time of the WBAN device and reduce the specific absorption rate. Due to near human transmission characteristics, WBAN device must strictly limit transmitting power.The power consumption of WBAN based of the ZigBee protocol is evaluated in Paper 3 , and the conclusion is that the protocol can not be used in WBAN if the power consumption of the ZigBee protocol is not improved.A dynamic scheduling strategy of Bluetooth for WBAN is proposed in Paper 4. The sensor nodes can achieve power saving by adaptively switching between the connection state and sleep state.

In addition to the above improved MAC protocol to Bluetooth and ZigBee technology, in recent years, the academic community has proposed a number of low-power MAC protocol specifically applied to the WBAN system, including CICADA and H-MAC ${ }^{[5]}$.CICADA is a MAC protocol for wireless multi-hop and mobile WBAN, which uses the method of real-time data transmission to improve the throughput and reduce transmission delay. H-MAC ${ }^{[6]}$ is a time division multiple access (TDMA) MAC layer protocol, combined with heart rate monitoring applications, sensor nodes automatically achieve time synchronization according to the heartbeat rhythm, and save power consumption for opening $\mathrm{RF}$ and receiving the time synchronization information.As the only published WBAN standards in the world, IEEE 802.15.6 proposes the power management strategy of WBAN from the macro and micro perspectives. Macro power management refers to the 
"hibernation": when a node is in a non-activated state in the non - Wake - up super frame, the node enters the "hibernation". Micro power management refers to the "dormancy": when the nodes are in the wake of the super frame and do not need to receive the beacon frame, the nodes in the beacon transmission time is not activated, that is to enter the "dormancy".

The above research shows: low power design idea should run through all aspects of WBAN system, from single layer protocol or transceiver design for power saving unable to obtain maximum power savings. Therefore, it is necessary to study the cross layer protocol, hardware and software optimization of low power design method.

\section{B. Security technology}

WBAN collected data is closely related with human physiological parameters, at the same time, WBAN data confidentiality is essential, because health monitoring data, which belong to personal privacy data, we must strictly limit the data which the only authorized users can access and use.The security requirement of WBAN is analyzed in Paper7, and the security of WBAN system is proposed, which is to ensure the confidentiality, integrity, fault tolerance and robustness of health information. Security attacks in WBAN can be divided into: 1) attacks on confidentiality and authentication, the attacker takes eavesdropping, spoofing and replay attacks; 2) attacks on the integrity, the attacker modifies the information content, causes error messages received; 3 ) attacks on the network availability, for example:attackers use denial of service to exhaust network resources.

Because the sensor nodes have strict low power consumption, it is very challenging to resist these attacks. If the use of complex security encryption measures, it is bound to lead to excessive energy consumption, and easy to affect the normal communication of sensor nodes, and then affect the patient's signs data collection.IEEE 802.15 .6 body area network standard defines the multi-level security level (level 0 2) communication, each security level is corresponding different protection levels and frame format $\left.{ }^{[8]}: 1\right)$ level 0 : insecure communication, the data is not certified in communication, also does not have the integrity protection; 2) level 1: only authentication, data transmission in the security authentication mode, but the data is not encrypted; 3) level 2: authentication and encryption. This is the communication mode of the highest level of security.Paper 9 also puts forward a lightweight security encryption mechanism which can effectively ensure the security of the data transmission, and has low complexity. Paper 10 proposes that the security of data transmission is ensured by using the security mechanism of cross layer protocol layer.

From the point of view of the current status of the research, the security and privacy research about WBAN communication must understand different types of application requirements of WBAN before the appropriate security mechanism is integrated. We must also consider the social, legal and regulatory constraints, and there is no full consideration of these factors in study on the existing WBAN.

\section{C. $Q o S$}

Existing QoS-guarantee mechanism for the traditional WSNs usually uses a single QoS parameters as optimization objectives. For example: reliability, latency, data rate, and mobility, it is not suitable for direct application to the WBAN. So Latre B has evaluated the reliability of CIADIA protocol, and proposed the improved mechanism which will further improve the transmission reliability. ${ }^{[11]}$ Zhou Gang studies the adaptive resource scheduling mechanism in WBAN, which ensures the reliable data transmission. ${ }^{[12]}$ Through evaluating the actual performance of WBAN prototype system receiver, Otto C performed a statistical analysis of the possible factors which causes network transmission reliability to decline. ${ }^{[13]}$ Medwin defines a variety of channel access methods: timing access, non timing access, temporary access and competitive access, for the different types, different QoS needs of the services. ${ }^{[14]}$

About the research of QoS security of WBAN, the above documents are the optimization method for single QoS performance index, and few studies on the joint 
optimization of the QoS performance index. WBAN system requirements of transmission reliability, energy efficiency, latency is higher than that of any previous communication system, these QoS performance indicators are equally important, if only for a single QoS parameters are optimized, often leads to a decrease in other QoS performance, is not conducive to the reliable and efficient transmission of medical services.Therefore, in order to guarantee the high quality transmission of data in WBAN, it is urgent to study the multi-objective optimization method based on multi-QoS performance index.

\section{WBAN STANDARD}

IEEE802.15 working group on early in 2007 on the established the TG 6 working group, to carry out the standardization of the WBAN, and released IEEE802.15.6 which is the world's first WBAN standard in March 2012. The Federal Communications Commission in February 2013 released the IEEE802.15.4J standard, the standard modeled on IEEE802.15.4 is defined in $236-2400 \mathrm{~m} \mathrm{~Hz}$ meet enhanced physical layer and MAC layer technology for the demand of medical service. In order to adapt to the characteristics of Chinese Medical band (174 -216, 407-425, 608-630MHz), IEEE 802.15 established TG4n work group, ${ }^{[15]}$ formulate the physical layer standard suitable for Chinese Medical band and corresponding MAC layer standard.China Communications Standards Association (CCSA) also in November 2012 propose the research project-WBAN communication technology requirements for medical and health applications, the goal is to develop standards for WBAN industry, which is applicable to China's medical and health applications.

In addition to the above newly developed WBAN standards, some mature wireless area network communication standard and wireless LAN standards also in a timely manner of evolution, in order to meet the networking applications (including the needs of WBAN applications), and strive to seize the market of short distance wireless communication. Bluetooth Technology Alliance in 2010 by the end of June launched flagship low-power Bluetooth version 4.0, the standard was strongly supported by the industry and Health Alliance has approved the Bluetooth 4.0 as the new standard of the low power consumption of LAN communication. In order to build the wisdom, the low power of the Internet of things, Bluetooth Technology Alliance in December 2013 officially released the Bluetooth core specification version of a Bluetooth 4.1 version. Bluetooth 4.1 version supports the coexistence of LTE and 3GPP, and enhance the data transmission rate, so as to improve the consumer experience. The protocol update can also enable the Bluetooth device to support a variety of roles, to support the innovation development of innovative developers, so as to consolidate the status of Bluetooth technology in the wireless Internet connection. WiFi alliance also launched for the development of the WLAN technology standards (802.11ah) for the Internet of things in 2010. The standard use of the below $1 \mathrm{GHz}$ free license band has greater coverage, support for more users, lower power consumption, for the low and medium speed optimization and enhancement, etc.

\section{WBAN INDUSTRIALIZATION}

In order to explore the advantages of WBAN technology in the field of medical and health care, the academic circle has made a lot of attempts on the application of WBAN, and various WBAN systems are emerging. Codeblue project of Harvard University based on TinyOS operating system developed a series hardware platforms for health monitoring, including wireless blood oxygen saturation monitoring equipment and wireless ECG monitoring equipment.European Mobi.Health project developed an end and end mobile health monitoring platform, through the universal mobile system and general packet radio service (GPRS) monitored on patients' physical signs dynamically.SMART projects researched and developed wireless vital signs monitoring system of emergency patients.Many domestic universities and research institutes, including the Chinese Academy of Sciences, Beijing Institute of Technology and Shanghai Jiao Tong University have developed a remote health monitoring system based on WBAN.In the industry, WNAB products have been widely used in the field of medical and health care.American Santiago hospital use WBAN products based 
on ZigBee technology to manage the hospital equipment, so that the use of equipment increased from $68 \%$ to $90 \%$, the loss rate dropped from $14 \%$ to 0 .

From the current situation of industrialization,potential of WBAN in the field of medical care has not been fully released.On the one hand, although there are several standards for WBAN, but because most of the standards are driven by a few companies or research institutions, intellectual property mastered in the hands of the individual units, lacking participation enthusiasm of full industry chain.On the other hand, the lack of support of the health care industry chain, relying on the efforts of a single link can not promote the wide application of WBAN technology .From the current situation, the promotion and application of WBAN in the medical field is not optimistic.In recent years WBAN has thrived in the field of consumer electronics, mainstream Internet company,Communications equipment company and Consumer electronics company released indigenous wearable devices, including all smart watches, bracelets, glasses and other. It can be predicted that WBAN will have a bright future.

\section{CONCLUSION}

In this paper, the key communication technology of WBAN and the status quo of industrialization are analyzed.From the research of WBAN communication technology, although the research on WBAN communication technology is endless, the WBAN technology is still immature,mainly reflected in the low power consumption, security and QoS, etc.,these factors have become the bottleneck which restrict the application and popularization of WBAN technology.From the WBAN standardization and industrialization of the status quo, the lack of strong products on the market, there is still a lot of space of large-scale industrial.There is still a lot of work to do in the future in the industrialization of WBAN technology, need the cooperation of whole industry chain of WBAN.

\section{REFERENCES}

[1] CHEN Wen .WBAN [J].ZTE Technology Journal,2014,20(6),57-59

[2] GAO Li ,WANG Ge.Study on Key Technology of Wireless Body Area
Network (WBAN) Application[J].Computer and Digital Engineering2013,41(2):327-329.

[3] Yu Kun-ming.Power Control of WBAN System WBAN[D].Xi'an Electronic and Science University,2013.DOI:10.7666/d.D365361.

[4] Wang Bao-zong.Bluetooth Technology and Application in WBAN[D].Southeast University.2010.

[5] YANG Song .Research on Multi Hop Routing Protocols in WBAN[D].Shanghai Jiao Tong University.2014

[6] Khaled A. Ali,Jahangir H. Sarker,Hussein T. Mouftah et al.A MAC protocol for Cognitive Wireless Body Area SensorNetworking[C].//6th ACM international wireless communications and mobile computing conference 2010. vol. 1.2010:168-172.

[7] XU Da-qing, YANG Yue-xiang .Application Research of Wireless Body Area Network Mobile Security Service[J].Computer Engineering and Design,2014,35(5):1593-1597

[8] 802.15.6-2012. IEEE Standard for local and metropolis an area net works - Pan 15.6: Wireless body a r ea net works[S].

[9] ZHANG Xiao-bo,YUAN Kai-guo.Research On Security Technology of WBAN[C].The academic annual meeting of the ninth session of the China Communications Society.2012:382-386.

[10] HE Ai-ling,.Research on WBAN Data Security Transmission Based on Physiological Characteristics[D]. Ningxia University,2014.

[11] Latre B,Braem B,Moerman I,et a1.A low - delay protocol for multiple wireless body area net works[C ]// Fourth Annual International conference on Mobile and Ubiquitous System s:Networking \& Services ,Philadelphia ,P A, 2007:1 - 8.

[12] Zhou Gang, Lu Jian, W an C Y ,et a1 BodyQoS: Adaptive and radio-agnostic QoS for body sensor net works[C ] / IEEE Infocom,2008 : $565-573$.

[13] Otto C , Milenkovic A,Sanders C , et a1. System architecture of a wireless body area senor network for ubiquitous health monitoring[J].Journal of Mobile M multimedia.2006.i(4) :307 -326.

[14] FENG Hui ,Research on reliable and efficient data transmission strategy in wireless body area network[D].University of Science \& Technology China.2014

[15] Physical layer utilizing dedicated medical bands in China[EB / OL].[2014- 01- 07]. HTTP://WWW.ieee802.or g/15/pub / TG4n.html. 